\title{
Design of Silent Actuators using Shape Memory Alloy
}

\author{
Jaideep Upadhyay ${ }^{1,2}$, Husain Khambati ${ }^{1,2}$, David Pinto ${ }^{1}$ \\ ${ }^{1}$ Benemérita Universidad Autónoma de Puebla, \\ Facultad de Ciencias de la Computación, Mexico \\ ${ }^{2}$ Manipal University Jaipur, India \\ \{jaideep1895@gmail.com, husainkhambati1895, davideduardopinto\}@gmail.com \\ http://www. Ike.buap.mx/
}

\begin{abstract}
In this paper we present a design for an actuator using a shape memory alloy called flexinol. This actuator can be used to mimic the movements of various muscle groups for robotic applications. This design takes advantage of the properties of shape memory alloys, especially flexinol and its high contraction force. The use of flexinol enables the device to be compact and be highly reliable. We also present an efficient way of controlling the movement of the shape memory alloy using pulse width modulation. The designs are to be embedded inside an artificial facial skin of a robot to expresses human like emotions in robots, in particular, as artificial muscle design for human cheek muscles and the muscles which are used only for linear movement of the human skin.
\end{abstract}

Keywords. Flexinol, shape memory alloy, actuators, artificial facial skin.

\section{Introduction}

Generating emotional facial expression in robots is a very challenging task which has been studied for different research teams. This interest has been motivated by the current progress of robots who has been evolved from only being useful in industrial settings to stay in close contact with human beings. In this way, emotion expression in robots is essential for human-robot interaction.

There have been developed different robots providing emotional expressions such as the ones introduced by the research team of Hiroshi Ishiguro. A number of realistic humanoid robots that include an adult woman and a child that can recite news reports in different languages and voices $[3,4]$.

There are, however, other research teams working in providing emotional expressions to realistic humanoids, for example, BINA48 [1], who is a humanoid robot created by Hanson Robotics and owned by Martine Rothblatt's Terasem Movement, Incorporated (TMI), which is a non-profit organization with the purpose of preserving, evoking, reviving and downloading human consciousness 
in non-biological or nantech bodies [2]. This robot was created with the aim of showing emotional expressions when having a dialog with human beings.

Some other devices exist around the world, however, we would like to talk about Arthur (see Figure 1), a humanoid robot capable of show emotional expressions in its face. This robot is held by the research team of the Language \& Knowledge Engineering Lab. at the Faculty of Computer Science of Benemerita Universidad Autonoma de Puebla.

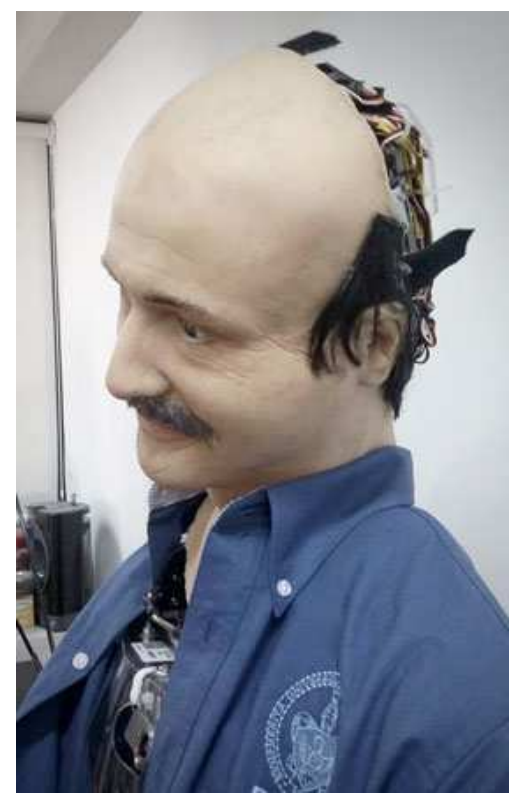

Fig. 1. Humanoid robot al LKE Laboratory.

Arthur has more than 20 servomotors under the skin which allows it to move its artificial skin in different positions with the aim of expressing emotions such as anger, happiness or sadness. Currently, this robot uses plastic strings in order to move the skin, which we consider to be an obsolete kind of technology. In this way, we would like to explore new ways to allow the humanoid robot to express those kind of emotions but with much more reliable technologies. This paper explores the use of new actuators based on Flexinol for such purpose.

The remaining of this paper is structured as follows. Section 2 motivates our research work presenting advantages of the approach. Section 3 discusses the design of actuators using shape memory alloy. Section 4 presents an analysis of the type of Flexinol wires employed in the experiments with different test over them. Section 5 show a preliminar design of the actuators proposed. Finally, in Section 6 , the conclusions of this research work are given. 


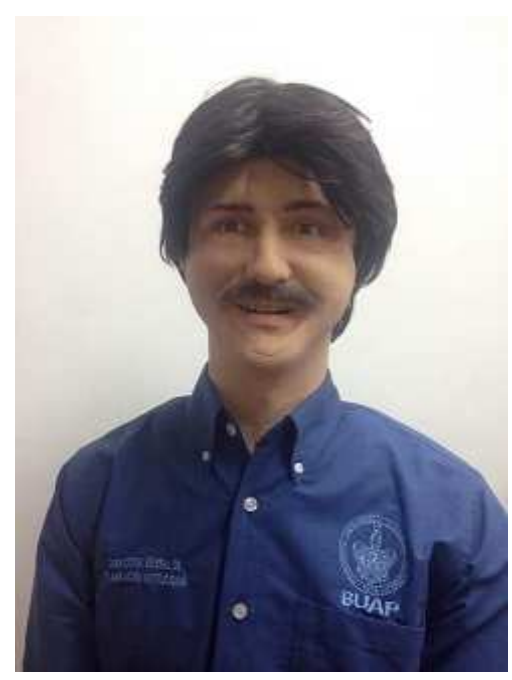

Fig. 2. Humanoid robot al LKE Laboratory.

\section{Motivation}

The current technology which deals with generation of facial expressions in a robot makes use of a complex placement of servo motors and links to impart expressions in the artificial skin of the robot. This system consumes a lot of power and is very complex to rectify if a problem arises. The strings or links are attached at various positions inside the artificial skin of the robot and to the rotating parts of the servo motors, this enables the servo motors to pull the skin from inside to mimic the human expressions. This method makes the skin of the robot very weak when the strings pull it and also damages the inside layer of the skin over a period of time. Due to the complex arrangement of the servo motors and the links inside, it is a great problem if a part is damaged or not working properly as the complexity of the system makes it impossible for the user to repair or replace the part on his own. The cost of the system also increases drastically as a lot of servo motors are used. Servos are bulky and due to their gearing system produce noise. Our method provides a simple solution of using Shape memory alloys as the primary actuator. The advantages of these alloys are abundant. They are smaller, lighter, easy to control, cheaper and reliable. The manufacturing of these actuators is also a simple process.

\section{Designing Silent Actuators}

The aim of this Research Project is to develop an actuator design which can replace servo motors and solenoids in robotic application. The design presented in the paper have several advantages over servo motors and solenoids as our design is smaller, less noisy and can be replaced very easily if damage occurs. 
As flexinol is basically just a wire so the designs can be configured to suit any situation very easily. Flexinol is a form of shape memory alloy as Shape memory alloys display two distinct crystal structures or phases. Temperature and internal crystal structure determine the phase that the Shape memory alloy will be at. Martensite exists at lower temperatures, and austenite exists at higher temperatures. When a Shape memory alloy is in martensite form at lower temperatures, the metal can easily be deformed into any shape. When the alloy is heated, it goes through transformation from martensite to austenite. In the austenite phase, the memory metal "remembers" the shape it had before it was deformed. Flexinols austenite phase is such that it allows the structure compresses. The flexinol wire contracts to upto $2 \%-5 \%$ of its original length when it is heated to austenite phase and thus produces a pulling force whose magnitude depends upon the diameter of the wire used. The heating of the flexinol is achieved by passing a certain amount of current through the wire. The flexinol wire inherently possesses very little resistance to the current applied, so a current limiting system is required to control the amount of current flowing through the wire otherwise this may cause irreparable damage to the structure of the alloy. Generally, the shape memory alloy has over a million life cycles but this depends on the stress exerted on the crystal structure of the alloy. High current, high temperatures or high loading on the wire may drastically affect its life cycles. The designs discussed in this paper will mainly focus on the artificial muscle design for human cheek muscles and the muscles which are used only for linear movement of the human skin.

\section{Analysis of Flexinol Wires}

For the efficient design of the actuators it was necessary to know the behaviour of the flexinol wires so various basic experiments were carried out.

Table 1 shows the specifications of three different flexinol wires as all the experiments were carried out using these wires only at room temperature.

\section{$4.1 \quad$ Test-1}

For the first test a 0.004-inch diameter wire was used having a phase transition temperature of $90 \mathrm{C}$ was used to determine the load bearing capacity of the wire. A current of 0.35 amps at a voltage of $5 \mathrm{~V}$ was supplied to the wire. The contracting force of the wire was measured to be $420 \mathrm{~g}$ approx. The life cycle of the wire was reduced to 10 cycles as the current supplied is approximately twice the amount of current for the safe operation of the wire as seen in the above table.

\section{$4.2 \quad$ Test-2}

For the second test a 0.015 -inch diameter wire was used having a phase transition temperature of $90 \mathrm{C}$ was used to determine the load bearing capacity of the wire. 
Table 1. Specifications of three different flexinol wires at room temperature.

\begin{tabular}{|l|c|c|c|}
\hline \multirow{2}{*}{ Feature } & \multicolumn{3}{|c|}{$\begin{array}{c}\text { Diameter Size } \\
\text { (Inches) }\end{array}$} \\
\cline { 2 - 4 } & $\mathbf{0 . 0 0 4}$ & $\mathbf{0 . 0 0 8}$ & $\mathbf{0 . 0 1 5}$ \\
\hline Resistance (Ohms/Inch) & 3.0 & 0.8 & 0.2 \\
Maximum Pull Force (grams) & 150 & 590 & 2,000 \\
Approximate* Current (mA) & 180 & 610 & 2,750 \\
Contraction* Time (seconds) & 1 & 1 & 1 \\
Off Time 90 C HT Wire** (seconds) & 0.4 & 2.2 & 10.0 \\
\hline
\end{tabular}

*Please note contraction time is directly related to electric current imposed. The guidelines are only approximations, since other factors like ambient temperature, air currents, and heat sinking will vary with specific devices.

** Approximate Cooling Time

A current of 2.91 amps at a voltage of $9 \mathrm{~V}$ was applied to the wire. The contracting force of the wire was measured to be $3 \mathrm{~kg}$ approx. The life cycle of the wire was reduced to 6-7 cycles and the cooling rate of the wire was also too low for rapid actuation.

\section{Preliminar Design of Silent Actuators}

In this paper we only sketch the general design of two actuators: a linear and a bending one.

The Linear Muscle Actuator should consist of two hollow shafts that should fit into one another. One shaft of the device must have a key and the other should have a keyway into which the keyed shaft will sit. This design is to prevent the two parts from twisting onto each other when it is actuated. A compression spring should be inserted into both the shafts. The flexinol wire has to be held using nuts and bolts on the outside of the structure so as to increase the cooling rate of the wire. The two flexinol wires should be connected in parallel in the circuit. The compression of the flexinol wire is expected to cause the two shafts to compress the spring and once the compression of the flexinol is removed the spring will allow the two shafts to return to their respective positions. When this device is going to be used as a facial muscle one end of the actuator should be fixed to a solid structure while the other end should be attached to the artificial skin in order to ensure the linear movement in one direction only. The amount of linear movement in this device shoulb be controlloed by an amount of current flowing through the flexinol wire by applying a PWM (pulse width modulation) signal of appropriate duty cycle.

The Bending Muscle Actuator should consist of a thin plastic plate shaped like a human facial muscle and a spring tightly attached at the middle of the top part of the plastic plate. The sole purpose of the plastic plate is to provide structure to the muscle actuator and to provide proper curvature while bending. Nuts and bolts are need for holding the flexinol wires (shape memory alloy, 
nitinol) in place and to also act as a medium of current flow in the wires. The flexinol wires should be wound between the nuts and bolts on either side of the plastic plate. The spring must be isolated from the nut and bolt assembly and the flexinol wires to stop the current flowing through the spring which can change the path of the current and the flexinol wires will fail to heat up and contract.

A spring having its compressed state as its natural state is going to be used to return the muscle actuator to its original position. The spring attached to the top part of the plastic plate will bend along with the plastic plate, thus causing an expansion in the spring, generating a contractive force in the spring and thus when the muscle actuator is switched off the spring will pull back the actuator to its original shape. The force exerted by the spring and the force exerted by the flexinol wire will act in opposite direction and the amount of bending will be controlled by varying the force exerted by the flexinol wire and thus different amount of bending can be achieved at different equilibrium points of the force exerted by the spring and the flexinol wire. The force exerted by the flexinol wire could be adjusted by varying the amount of current supplied to the it. The force exerted by the flexinol wire will bend the plastic plate and it will mimic the motion of a muscle.

\section{Conclusions and Further Work}

In this paper we have presented a general desing of silent actuators for a humanoid robot with the aim of having in a near future new technology for expressing emotions in this robot using silent and much more reliable actuators.

Some of the conclusions after analyzing the wire proposed to use follows:

1. Use of a current limiting circuit is a must as the wire tends to draw more current than its rated value. The greater the current drawn the greater the temperature in the wire which will cause a decrease in the life cycles of the shape memory alloy.

2. We would prefer thinner wires to their thicker counterparts. The thicker the wire the more the power is required for its actuation and also the cooling time of the wire is increased drastically. If the force required by these flexinol wires is greater, then a bundle of thinner wires should be preferred over the thicker wires.

Hence from the conclusions of the above experiments the 0.004-inch wire was used as an actuator wire for the artificial muscles as it has a very quick cooling rate thus a very good reaction time and a current limiting circuit was installed to keep the current of the wire below the rated current. The thicker wires would require a separate system for cooling which would increase the size of our device.

Our future work will involve the designing of the complete facial muscle groups. Also it will involve designing of the skeletal frame and skin for the proper actuation of the muscle groups. Designing of the complete control system for the entire facial expression system will also be a priority in future work. Testing and 
implementation of better grade quality of shape memory alloys or other such smart materials in the design for better efficiency and control.

\section{References}

1. Harmon, A.: Making friends with a robot named bina48. New York Times, The address of the publisher (July 4th)

2. Inc., T.M.: Terasem movement: Lives are good. http://www.terasemcentral.org/ (2016), retrieved October 10, 2016

3. Nishio, S., Ishiguro, H., Hagita, N.: Geminoid: Teleoperated android of an existing person. In: de Pina Filho, A.C. (ed.) Humanoid Robots: New Developments, pp. 343-352. I-Tech Education and Publishing, Vienna, Austria (Jun 2007), http://www.intechopen.com/articles/show/title/geminoid_ -teleoperated_android_of_an_existing_person

4. Nishio, S., Taura, K., Ishiguro, H.: Regulating emotion by facial feedback from teleoperated android robot. In: International Conference on Social Robotics. pp. 388-397. Chengdu, China (Oct 2012), http://link.springer.com/chapter/10. 1007/978-3-642-34103-8_39 